International Journal of Bifurcation and Chaos, Vol. 20, No. 1 (2010) 81-97

(C) World Scientific Publishing Company

DOI: $10.1142 / \mathrm{S} 0218127410025429$

\title{
SYNCHRONIZATION OF CHAOTIC FRACTIONAL-ORDER SYSTEMS VIA LINEAR CONTROL
}

\author{
ZAID M. ODIBAT* \\ Faculty of Science and Technology, University of Le Havre, \\ 76058 Le Havre Cedex, France \\ z.odibat@gmail.com \\ NATHALIE CORSON ${ }^{\dagger}$ and M. A. AZIZ-ALAOUI ${ }^{\ddagger}$ \\ Appl. Math. Lab., University of Le Havre, 76058, \\ Le Havre Cedex, France \\ ${ }^{\dagger}$ nathalie.corson@univ-lehavre.fr \\ ${ }^{\ddagger}$ aziz.alaoui@univ-lehavre.fr \\ CYRILLE BERTELLE \\ LITIS, University of Le Havre, \\ 76058 Le Havre Cedex, France \\ cyrille.bertelle@univ-lehavre.fr
}

Received February 25, 2008; Revised May 11, 2009

\begin{abstract}
The chaotic dynamics of fractional-order systems has attracted much attention recently. Chaotic synchronization of fractional-order systems is further studied in this paper. We investigate the chaos synchronization of two identical systems via a suitable linear controller applied to the response system. Based on the stability results of linear fractional-order systems, sufficient conditions for chaos synchronization of these systems are given. Control laws are derived analytically to achieve synchronization of the chaotic fractional-order Chen, Rössler and modified Chua systems. Numerical simulations are provided to verify the theoretical analysis.
\end{abstract}

Keywords: Chaos synchronization; fractional-order system; Caputo fractional derivative; MittagLeffler function; stability; linear control.

\section{Introduction}

Chaos, as an important topic in nonlinear science, has been investigated and studied in mathematical and physical communities in the last few decades and research efforts have been focused on chaos control and chaos synchronization problems in many dynamical systems. During the last two decades, synchronization in chaotic dynamical systems has attracted a great deal of attention [Yamada \& Fujisaka, 1983; Pecora \& Carrol, 1990; Ott et al., 1990; Chen \& Dong, 1998; Derivière \& Aziz-Alaoui, 2003; Aziz-Alaoui, 2006] since the seminal papers by Yamada and Fujisaka [1983] and Pecora and Carrol [1990]. An approach to the study of synchronization is to use a controller for the output of the slave (reponse) system to copy the master (drive)

\footnotetext{
*On sabbatical leave from Prince Abdullah Bin Ghazi Faculty of Science and IT, Al-Balqa’ Applied University, Salt-Jordan.

${ }^{\ddagger}$ Author for correspondence
} 
system. On the other hand, the development of models based on fractional-order differential systems has recently gained popularity in the investigation of dynamical systems. Fractional derivatives provide an excellent instrument to describe memory and hereditary properties of various materials and processes. The main reason for using the integer-order models was the absence of solution methods for fractional differential equations. The advantages or the real objects of the fractionalorder systems [Petráš, 2008] are that we have more degrees of freedom in the model and that a "memory" is included in the model (fractional-order systems have an unlimited memory).

Recently, studying fractional-order systems has become an active research area. The chaotic dynamics of fractional-order systems began to attract much attention in recent years [Podlubny, 1999; Hilfer, 2000]. It has been shown that fractionalorder systems, as generalizations of many wellknown systems, can also behave chaotically, such as the fractional Duffing system [Ge \& Ou, 2007], the fractional Chua system [Petráš, 2008; Hartley et al., 1995], the fractional Rössler system [Li \& Chen, 2004a], the fractional Chen system [Li \& Peng, 2004; Li \& Chen, 2004b; Lu \& Chen, 2006], the fractional Lorenz system [Grigorenko \& Grigorenko, 2003], the fractional Arneodo system [Arneodo et al., 1985] and the fractional Lü system [Deng \& Li, 2005]. In [Hartley et al., 1995; Li \& Chen, 2004a, 2004b; Li \& Peng, 2004] it has been shown that some fractional-order systems can produce chaotic attractors with order less than 3 .

Recent studies show that chaotic fractionalorder systems can also be synchronized [Lu \& Chen, 2006; Deng \& Li, 2005; Li et al., 2003; Li \& Zhou, 2005; Zhou et al., 2008; Peng, 2007]. In many literatures, synchronization among fractional-order systems is only investigated through numerical simulations. A simple method to synchronize chaotic fractional-order systems based on the stability criteria of linear differential systems, see [Matignon, 1996; Deng et al., 2007], is presented in [Sheu et al., 2007; Yan \& Li, 2007; Li \& Yan, 2007]. Numerical algorithms for chaos synchronization of fractionalorder systems based on Laplace transform theory are presented in [Wang et al., 2006; Li et al., 2006; Zhu et al., 2009; Wu et al., 2008; Yu \& Li, 2008].

In this paper, we recall and present some stability results for linear fractional-order systems. Then, based on these results, we study the chaos synchronization of fractional-order systems. Using the drive-response concept, a linear feedback control law is derived to achieve synchronization of the two chaotic fractional-order systems. The synchronization controllers are investigated theoretically and numerical simulations are then presented in the case of fractional-order Chen, Rössler and modified Chua systems.

\section{Preliminaries and Definitions}

There are several definitions of a fractional derivative of order $\alpha>0$ [Podlubny, 1999; Caputo, 1967]. The two most commonly used are the RiemannLiouville and Caputo definitions. Each definition uses Riemann-Liouville fractional integration and derivatives of whole order. The difference between the two definitions appears in the order of evaluation. The Riemann-Liouville fractional integral operator of order $\alpha \geq 0$ of the function $f(t)$ is defined as,

$$
J^{\alpha} f(t)=\frac{1}{\Gamma(\alpha)} \int_{0}^{t}(t-\tau)^{\alpha-1} f(\tau) d \tau, \quad t>0 .
$$

Some properties of the operator $J^{\alpha}$ can be found, for example, in [Podlubny, 1999; Gorenflo \& Mainardi, 1997; Oldham \& Spanier, 1974]. We recall only the two following properties. For $\mu \geq-1, \alpha, \beta \geq 0$ and $\gamma>-1$, we have,

$$
\begin{aligned}
J^{\alpha} J^{\beta} f(t) & =J^{\alpha+\beta} f(t), \\
J^{\alpha} t^{\gamma} & =\frac{\Gamma(\gamma+1)}{\Gamma(\alpha+\gamma+1)} t^{\alpha+\gamma} .
\end{aligned}
$$

In this study, Caputo definition is used and the fractional derivative of $f(t)$ is defined as, $J^{m-\alpha} D^{m} f(t)$,

$$
D^{\alpha} f(t)=\frac{1}{\Gamma(m-\alpha)} \int_{0}^{t} \frac{f^{(m)}(\tau)}{(t-\tau)^{\alpha-m+1}} d \tau,
$$

for $m-1<\alpha \leq m, m \in \mathbb{N}, t>0$.

Caputo definition has the advantage of dealing properly with initial value problems in which the initial conditions are given in terms of the field variables with their integer order which is the case in most physical processes. Fortunately, the Laplace transform of the Caputo fractional derivative satisfies,

$$
L\left\{D^{\alpha} f(t)\right\}=s^{\alpha} L\{f(t)\}-\sum_{k=0}^{m-1} f^{(k)}\left(0^{+}\right) s^{\alpha-1-k},
$$

where $m-1<\alpha \leq m$. 
The Laplace transform of Caputo fractional derivative requires the knowledge of the (bounded) initial values of the function and of its integer derivatives of order $k=1,2, \ldots, m-1$. It has been found that the solution of many fractionalorder differential equations can be written in terms of Mittag-Leffler functions, which are relevant for their connection with fractional calculus. The oneparameter Mittag-Leffler function $E_{\alpha}(z)$ is defined in [Podlubny, 1999],

$$
E_{\alpha}(z)=\sum_{k=0}^{\infty} \frac{z^{k}}{\Gamma(\alpha k+1)}, \quad \alpha>0, \quad z \in \mathrm{C},
$$

which is an entire function. The Laplace transform for $E_{\alpha}(z)$ is given by [Podlubny, 1999],

$$
L\left\{E_{\alpha}\left(-\lambda t^{\alpha}\right)\right\}=\frac{s^{\alpha-1}}{s^{\alpha}+\lambda} .
$$

The Mittag-Leffler function provides a simple generalization of the exponential function. For studying the stability of fractional order systems, we need the final value theorem.

Theorem 1. Let $F(s)$ be the Laplace transform of function $f(t)$. If all poles of $s F(s)$ are in the open left half plane, then,

$$
\lim _{t \rightarrow \infty} f(t)=\lim _{s \rightarrow 0} s F(s) .
$$

The final value theorem gives information about the asymptotic behavior of $f(t)$ (how $f(t)$ behaves as $t \rightarrow+\infty)$ directly from $F(s)$. This result plays an important role in the derivation of the linear feedback control laws to achieve synchronization of the chaotic fractional-order systems.

\section{Stability Results of Linear Fractional Systems}

Stability of linear fractional systems, which is of main interest in control theory, has been thoroughly investigated where necessary and sufficient conditions have been derived, see [Matignon, 1996; Deng et al., 2007] or references therein. Matignon [1996] introduced the stability properties for some linear fractional-order systems. Deng et al. [2007] studied the stability of $n$-dimensional linear fractional differential equations with time delays. In this section, we recall the main stability properties and then, we present a new property for a general class of fractional-order systems. For this object, we consider the following linear system of fractional differential equations,

$$
\frac{d^{\alpha}}{d t^{\alpha}} \mathbf{x}(t)=A \mathbf{x}(t), \quad \mathbf{x}(0)=\mathbf{x}_{0}
$$

where $\mathbf{x} \in \mathbb{R}^{n}$, the matrix $A \in \mathbb{R}^{n} \times \mathbb{R}^{n}, \alpha=$ $\left[\alpha_{1}, \alpha_{2}, \ldots, \alpha_{n}\right]$ indicates the fractional orders, $d^{\alpha} / d t^{\alpha}=\left[\left(d^{\alpha_{1}} / d t^{\alpha_{1}}\right),\left(d^{\alpha_{2}} / d t^{\alpha_{2}}\right), \ldots,\left(d^{\alpha_{n}} / d t^{\alpha_{n}}\right)\right]$ and $d^{\alpha_{i}} / d t^{\alpha_{i}}$ is the Caputo fractional derivative of order $\alpha_{i}$, where $0<\alpha_{i} \leq 1$ for $i=1,2, \ldots, n$. If $\alpha=\alpha_{1}=\alpha_{2}=\cdots=\alpha_{n}$, then the stability of the fractional-order system (7) has been studied in [Matignon, 1996], where necessary and sufficient conditions have been introduced. The following stability result was derived in [Matignon, 1996].

Theorem 2. The autonomous fractional-order system (7), when $\alpha=\alpha_{1}=\alpha_{2}=\cdots=\alpha_{n}$, is asymptotically stable iff $|\arg (\operatorname{spec}(A))|>\alpha \pi / 2$. In this case the components of the state decay towards 0 like $t^{-\alpha}$.

When $\alpha=1$, the stability occurs if no poles of the linear system (7) lie in the closed right half-plane of the Laplace plane which agrees with well-known results for ordinary linear differential systems.

If $\alpha_{1}, \alpha_{2}, \ldots, \alpha_{n}$ are rational positive numbers, then we have the following result, which is introduced in [Deng et al., 2007].

Theorem 3. Suppose that $\alpha_{i}$ 's are rational numbers between 0 and 1 , for $i=1,2, \ldots, n$. Let $\gamma=$ $1 / m$ where $m$ is the least common multiple of the denominators $m_{i}$ of $\alpha_{i}$ 's, where $\alpha_{i}=k_{i} / m_{i}$, $k_{i}, m_{i} \in \mathbb{N}, i=1,2, \ldots, n$. Then system ( 7$)$ is asymptotically stable if all roots $\lambda$ of the equation $\operatorname{det}\left(\operatorname{diag}\left(\lambda^{m \alpha_{1}}, \lambda^{m \alpha_{2}}, \ldots, \lambda^{m \alpha_{n}}\right)-A\right)=0$ satisfy $|\arg (\lambda)|>\gamma \pi / 2$.

Now, if $\alpha_{1}, \alpha_{2}, \ldots, \alpha_{n}$ are any positive numbers between 0 and 1 , not necessary equals or rational numbers, then we have the following new result.

Theorem 4. The autonomous fractional-order system ( 7$)$ is asymptotically stable if $|\arg (\operatorname{spec}(A))|>$ $\pi / 2$, that is $a_{11}, a_{22}, \ldots, a_{n n}<0$, when $A$ is an upper or lower triangular matrix.

Proof. For simplicity, we give the proof when $A$ is an $3 \times 3$ lower triangular matrix. A similar way can be followed to prove the general theorem. Consider 
the fractional-order system,

$$
\left(\begin{array}{l}
\frac{d^{\alpha_{1}} x}{d t^{\alpha_{1}}} \\
\frac{d^{\alpha_{2}} y}{d t^{\alpha_{2}}} \\
\frac{d^{\alpha_{3}} z}{d t^{\alpha_{3}}}
\end{array}\right)=\left(\begin{array}{ccc}
a_{11} & 0 & 0 \\
a_{21} & a_{22} & 0 \\
a_{31} & a_{32} & a_{33}
\end{array}\right)\left(\begin{array}{l}
x \\
y \\
z
\end{array}\right)
$$

Applying Laplace transform to system (8), letting $X(s)=L((x(t)), Y(s)=L(y(t)), Z(s)=L(z(t))$ and using formula (3), we obtain,

$$
\left\{\begin{aligned}
s^{\alpha_{1}} X(s)-s^{\alpha_{1}-1} x(0) & =a_{11} X(s) \\
s^{\alpha_{2}} Y(s)-s^{\alpha_{2}-1} y(0) & =a_{21} X(s)+a_{22} Y(s) \\
s^{\alpha_{3}} Z(s)-s^{\alpha_{3}-1} z(0)= & a_{31} X(s)+a_{32} Y(s) \\
& +a_{33} Z(s)
\end{aligned}\right.
$$

It follows from Eqs. (9) that,

$$
\left\{\begin{aligned}
X(s)= & \frac{s^{\alpha_{1}-1} x(0)}{s^{\alpha_{1}}-a_{11}} \\
Y(s)= & \frac{s^{\alpha_{2}-1} y(0)}{s^{\alpha_{2}}-a_{22}}+\frac{a_{21} s^{\alpha_{1}-1} x(0)}{\left(s^{\alpha_{1}}-a_{11}\right)\left(s^{\alpha_{2}}-a_{22}\right)} \\
Z(s)= & \frac{s^{\alpha_{3}-1} z(0)}{s^{\alpha_{3}}-a_{33}}+\frac{a_{31} s^{\alpha_{1}-1} x(0)}{\left(s^{\alpha_{1}}-a_{11}\right)\left(s^{\alpha_{3}}-a_{33}\right)} \\
& +\frac{a_{32} s^{\alpha_{2}-1} y(0)}{\left(s^{\alpha_{2}}-a_{22}\right)\left(s^{\alpha_{3}}-a_{33}\right)} \\
& +\frac{a_{21} a_{32} s^{\alpha_{1}-1} x(0)}{\left(s^{\alpha_{1}}-a_{11}\right)\left(s^{\alpha_{2}}-a_{22}\right)\left(s^{\alpha_{3}}-a_{33}\right)}
\end{aligned}\right.
$$

In particular, using (5), we have $x(t)=x(0)$ $E_{\alpha_{1}}\left(a_{11} t^{\alpha_{1}}\right)$, where $E_{\alpha}(t)$ is the Mittag-Leffler function. From the properties of this function, see [Gorenflo \& Mainardi, 1997], we can conclude that $x(t) \rightarrow 0$ as $t \rightarrow+\infty$ if $a_{11}<0$. In view of (10), all poles of $s X(s), s Y(s)$ and $s Z(s)$ lie in the open left half-plane if $a_{11}, a_{22}, a_{33}<0$. Hence, using the final value theorem we get,

$$
\left\{\begin{array}{l}
\lim _{t \rightarrow \infty} x(t)=\lim _{s \rightarrow 0} s X(s)=0, \\
\lim _{t \rightarrow \infty} y(t)=\lim _{s \rightarrow 0} s Y(s)=0, \\
\lim _{t \rightarrow \infty} z(t)=\lim _{s \rightarrow 0} s Z(s)=0 .
\end{array}\right.
$$

As a result, the states $x(t), y(t), z(t)$ decay towards 0 , as $t \rightarrow+\infty$, if $a_{11}, a_{22}, a_{33}<0$. Therefore, in this case, system (8) is asymptotically stable.

\section{Synchronization of Fractional-Order Systems}

In this section, based on the stability results of fractional differential equations, we briefly discuss the issue of controlling fractional-order chaotic systems to realize synchronization with linear error feedback control. In order to observe synchronization behavior, we construct the master (drive) system and the slave (response) system as,

$$
\begin{aligned}
& \operatorname{Master}\left\{\begin{aligned}
\frac{d^{\alpha_{1}} x_{m}}{d t^{\alpha_{1}}}= & b_{11} x_{m}+b_{12} y_{m}+b_{13} z_{m} \\
& +g_{1}\left(x_{m}, y_{m}, z_{m}, t\right) \\
\frac{d^{\alpha_{2}} y_{m}}{d t^{\alpha_{2}}}= & b_{21} x_{m}+b_{22} y_{m}+b_{23} z_{m} \\
& +g_{2}\left(x_{m}, y_{m}, z_{m}, t\right) \\
\frac{d^{\alpha_{3}} z_{m}}{d t^{\alpha_{3}}}= & b_{31} x_{m}+b_{32} y_{m}+b_{33} z_{m} \\
& +g_{3}\left(x_{m}, y_{m}, z_{m}, t\right)
\end{aligned}\right. \\
& \text { Slave }\left\{\begin{aligned}
\frac{d^{\alpha_{1}} x_{s}}{d t^{\alpha_{1}}}= & b_{11} x_{s}+b_{12} y_{s}+b_{13} z_{s} \\
& +g_{1}\left(x_{m}, y_{m}, z_{m}, t\right)+u_{1}(t) \\
\frac{d^{\alpha_{2}} y_{s}}{d t^{\alpha_{2}}}= & b_{21} x_{s}+b_{22} y_{s}+b_{23} z_{s} \\
& +g_{2}\left(x_{m}, y_{m}, z_{m}, t\right)+u_{2}(t) \\
\frac{d^{\alpha_{3}} z_{s}}{d t^{\alpha_{3}}}= & b_{31} x_{s}+b_{32} y_{s}+b_{33} z_{s} \\
& +g_{3}\left(x_{m}, y_{m}, z_{m}, t\right)+u_{3}(t)
\end{aligned}\right.
\end{aligned}
$$

where $d^{\alpha_{i}} / d t^{\alpha_{i}}$ is the fractional differential operator in Caputo sense, $0<\alpha_{i} \leq 1, g_{i}$ is a nonlinear function and $b_{i j}$ are real constants, for $i, j=$ $1,2,3$. Subscripts $m$ and $s$ stand for the master system and slave system, respectively, $\mathbf{u}(t)=$ $\left[u_{1}(t), u_{2}(t), u_{3}(t)\right]^{T}$ is the linear controller to be designed such that these two chaotic systems synchronize. Defining the synchronization error as,

$$
\begin{aligned}
& e_{1}(t)=x_{s}(t)-x_{m}(t) \\
& e_{2}(t)=y_{s}(t)-y_{m}(t) \\
& e_{3}(t)=z_{s}(t)-z_{m}(t)
\end{aligned}
$$


we get the error system,

$$
\left\{\begin{array}{l}
\frac{d^{\alpha_{1}} e_{1}}{d t^{\alpha_{1}}}=b_{11} e_{1}+b_{12} e_{2}+b_{13} e_{3}+u_{1}(t) \\
\frac{d^{\alpha_{2}} e_{2}}{d t^{\alpha_{2}}}=b_{21} e_{1}+b_{22} e_{2}+b_{23} e_{3}+u_{2}(t) \\
\frac{d^{\alpha_{3}} e_{3}}{d t^{\alpha_{3}}}=b_{31} e_{1}+b_{32} e_{2}+b_{33} e_{3}+u_{3}(t)
\end{array}\right.
$$

Our aim is to determine the controller $\mathbf{u}(t)$, given below, such that the drive system (12) and the response system (13) are synchronized $(\|\mathbf{e}(t)\| \rightarrow 0$, as $t \rightarrow+\infty)$.

$$
\begin{aligned}
\mathbf{u}(t) & =\left(\begin{array}{l}
u_{1}(t) \\
u_{2}(t) \\
u_{3}(t)
\end{array}\right) \\
& =\left(\begin{array}{lll}
a_{11} & a_{12} & a_{13} \\
a_{21} & a_{22} & a_{23} \\
a_{31} & a_{32} & a_{33}
\end{array}\right) \cdot\left(\begin{array}{l}
e_{1}(t) \\
e_{2}(t) \\
e_{3}(t)
\end{array}\right)
\end{aligned}
$$

where $a_{i j}$ are real constants. For this purpose, we design the controller $\mathbf{u}(t)=\left[u_{1}(t), u_{2}(t), u_{3}(t)\right]^{T}$ such that the solutions of the error system $e_{i}(t)$ go to $0, i=1,2,3$, as $t$ goes to $+\infty$. In this paper, three cases are studied to design the controller $\mathbf{u}(t)$.

Case 1. If $\alpha=\alpha_{1}=\alpha_{2}=\alpha_{3}$ then, using Theorem 2 , the drive system (12) and the response system $(13)$ are synchronized when $\left|\arg \left(\operatorname{spec}\left(A_{u}\right)\right)\right|>$ $\alpha \pi / 2$, where,

$$
A_{u}=\left(\begin{array}{lll}
a_{11}+b_{11} & a_{12}+b_{12} & a_{13}+b_{13} \\
a_{21}+b_{21} & a_{22}+b_{22} & a_{23}+b_{23} \\
a_{31}+b_{31} & a_{32}+b_{32} & a_{33}+b_{33}
\end{array}\right)
$$

In this case, we choose the controller $\mathbf{u}(t)$ such that $|\arg (\lambda)|>\alpha \pi / 2$ for every eigenvalue $\lambda$ of the matrix $A_{u}$. Of course, if all eigenvalues of $A_{u}$ are negative real numbers then both systems achieve synchronization.

Case 2. If $\alpha_{i}$ 's are rational numbers such that $\alpha_{i}=k_{i} / m_{i}, \gamma=1 / m$ where $m$ is the least common multiple of the denominators $m_{i}$ of $\alpha_{i}$ 's, $k_{i}, m_{i} \in \mathbb{N}$, for $i=1,2,3$, then, using Theorem 3 , the drive system (12) and the response system (13) are synchronized when all roots $\lambda$ of the characteristic equation $\operatorname{det}\left(\operatorname{diag}\left(\lambda^{m \alpha_{1}}, \lambda^{m \alpha_{2}}, \lambda^{m \alpha_{3}}\right)-A_{u}\right)=0$ satisfy $|\arg (\lambda)|>\gamma \pi / 2$. In this case, we choose the controller $\mathbf{u}(t)$ such that all the roots of the equation $\operatorname{det}\left(\operatorname{diag}\left(\lambda^{r_{1}}, \lambda^{r_{2}}, \lambda^{r_{3}}\right)-A_{u}\right)=0$ satisfy $|\arg (\lambda)|>$ $\gamma \pi / 2$, where $r_{i}=m k_{i} / m_{i}$.
Case 3. In particular, if $\alpha_{1}, \alpha_{2}, \alpha_{3}$ are any positive real numbers between 0 and 1 then, using Theorem 4, the drive system (12) and the response system (13) are synchronized when $A_{u}$ is an upper or lower triangular matrix and all eigenvalues of $A_{u}$ are negative real numbers. In this case, we choose the controller $\mathbf{u}(t)$ such that $A_{u}$ becomes an upper or lower triangular matrix and all roots of the characteristic equation $\left(\lambda-a_{11}-b_{11}\right)\left(\lambda-a_{22}-b_{22}\right)(\lambda-$ $\left.a_{33}-b_{33}\right)=0$ are negative real numbers.

To demonstrate these techniques, some examples of synchronization for two identical fractionalorder chaotic systems are discussed in the following sections.

\section{Synchronization of Fractional-Order Chen Systems}

Here, we consider Chen system, which has been found by Chen and Ueta [1999] in 1999. Its fractional version reads as,

$$
\left\{\begin{array}{l}
\frac{d^{\alpha_{1}} x}{d t^{\alpha_{1}}}=a(y-x) \\
\frac{d^{\alpha_{2}} y}{d t^{\alpha_{2}}}=(c-a) x-x z+c y \\
\frac{d^{\alpha_{3}} z}{d t^{\alpha_{3}}}=x y-b z
\end{array}\right.
$$

where $\alpha=\left(\alpha_{1}, \alpha_{2}, \alpha_{3}\right)$ is subject to $0<\alpha_{1}, \alpha_{2}, \alpha_{3} \leq$ 1. When $\alpha=(1,1,1)$, system (17) is the original integer order Chen system, which is chaotic, for example, when $(a, b, c)=(35,3,28)$, see [Chen \& Ueta, 1999]. Simulations are performed to obtain chaotic behavior of the fractional-order Chen system for different fractional orders $\alpha$. Simulation results demonstrate that chaos indeed exists in the fractional-order Chen system with order less than 3 (i.e. $\alpha_{1}+\alpha_{2}+\alpha_{3}<3$ ). For example, chaotic attractors are found in [Yan \& Li, 2007] when $\alpha=(0.95,0.95,0.95)$ and $(a, b, c)=$ $(35,3,28)$. In [Li \& Chen, 2004b] chaotic behaviors are found when $\alpha=(0.9,0.9,0.9)$ and $(a, b, c)=$ $(35,3,28)$.

Moreover, in [Wang et al., 2006], it is found that for the parameters $\alpha=(0.985,0.99,0.98)$ and $(a, b, c)=(35,3,28)$ the fractional-order Chen system can display chaotic attractors. The chaotic attractors of fractional Chen system (17) for some fractional orders are shown in Figs. 1-4. 

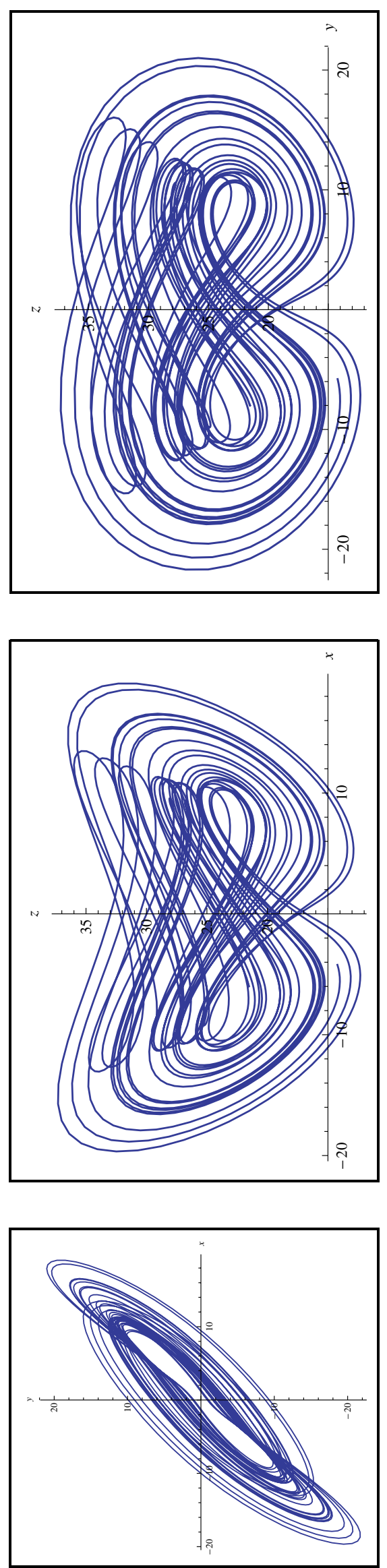

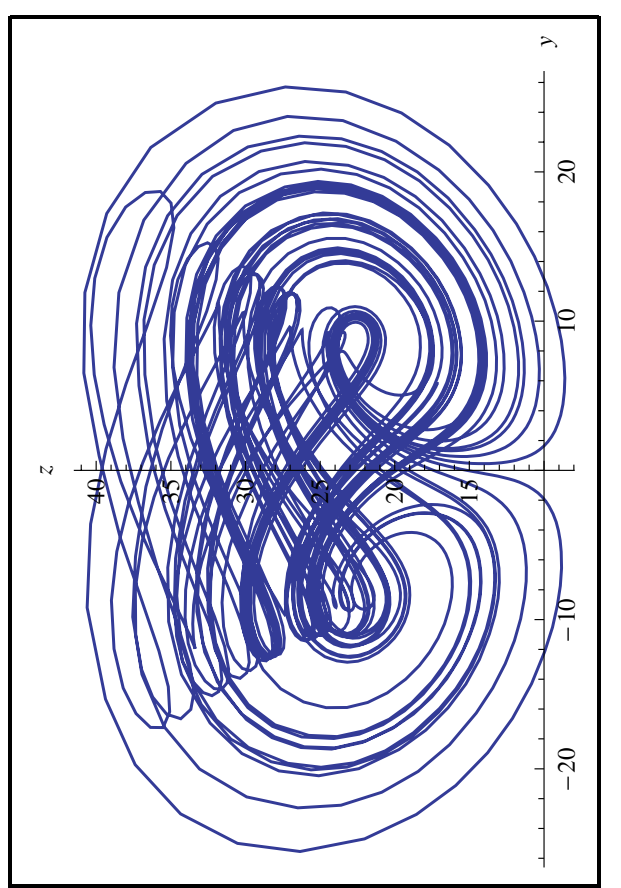

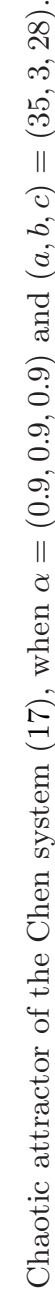

它

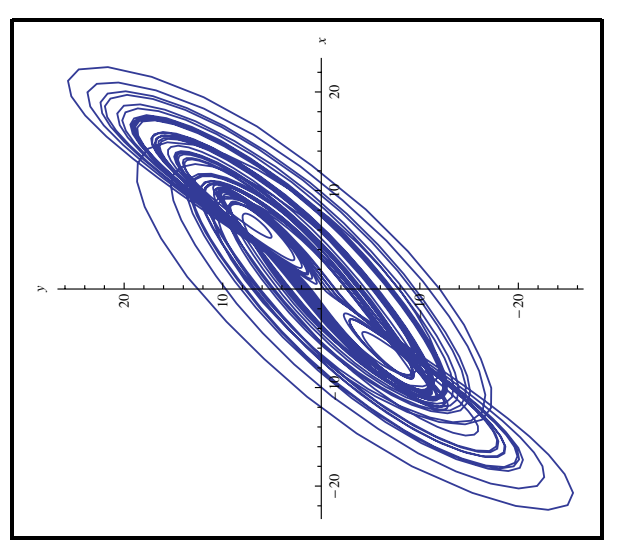



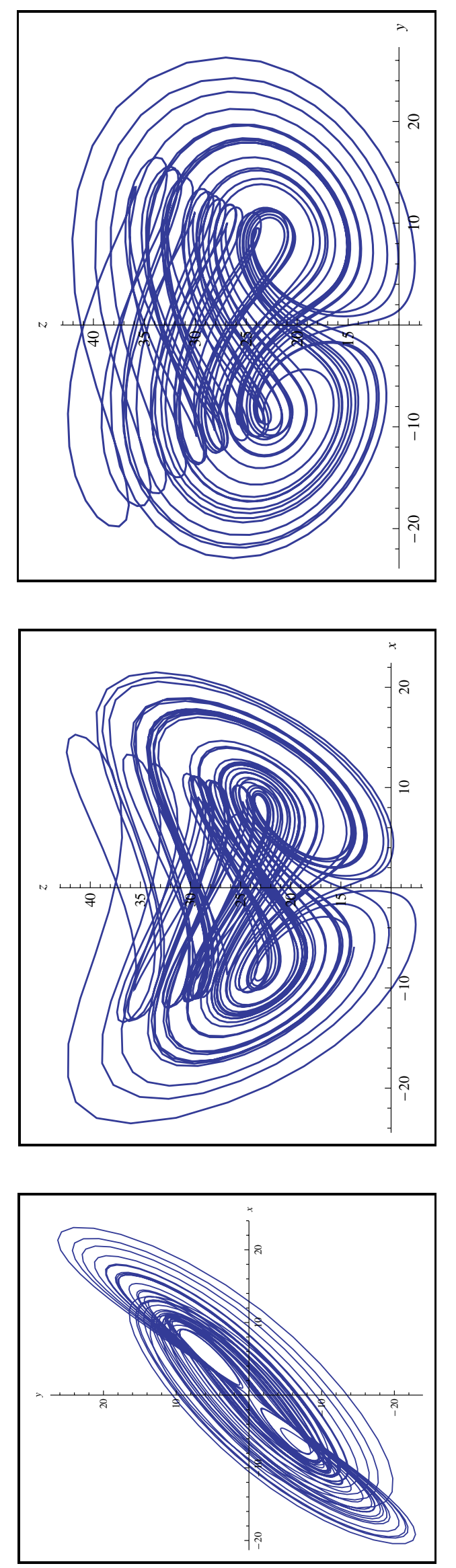

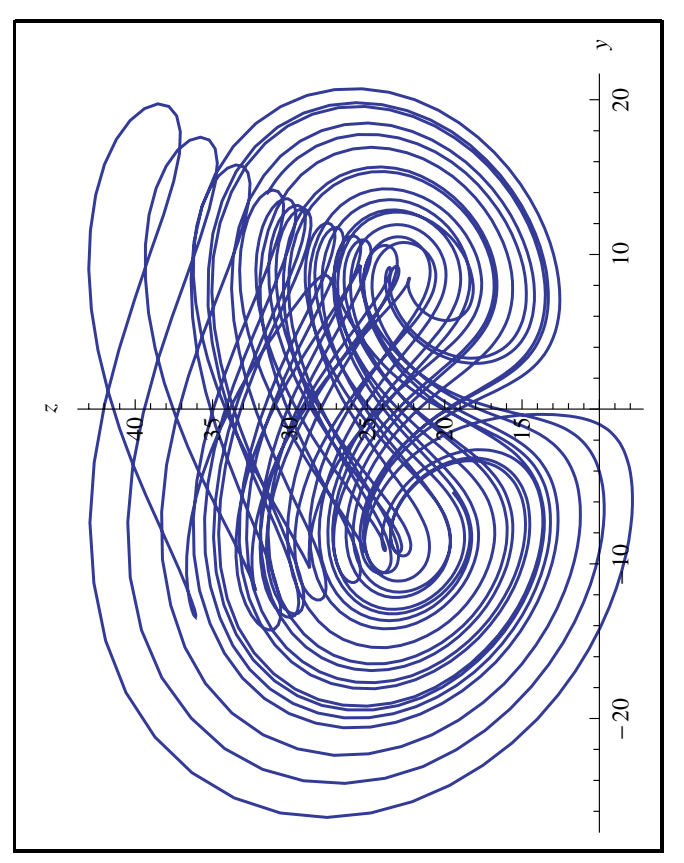

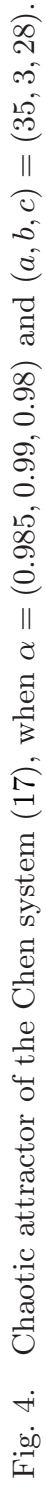


According to our approach, the drive and the response fractional-order Chen systems are described as,

$$
\begin{aligned}
& \left(\begin{array}{l}
\frac{d^{\alpha_{1}} x_{m}}{d t^{\alpha_{1}}} \\
\frac{d^{\alpha_{2}} y_{m}}{d t^{\alpha_{2}}} \\
\frac{d^{\alpha_{3}} z_{m}}{d t^{\alpha_{3}}}
\end{array}\right)=\left(\begin{array}{ccc}
-a & a & 0 \\
c-a & c & 0 \\
0 & 0 & -b
\end{array}\right)\left(\begin{array}{l}
x_{m} \\
y_{m} \\
z_{m}
\end{array}\right) \\
& +\left(\begin{array}{c}
0 \\
-x_{m} z_{m} \\
x_{m} y_{m}
\end{array}\right) \\
& \left(\begin{array}{l}
\frac{d^{\alpha_{1}} x_{s}}{d t^{\alpha_{1}}} \\
\frac{d^{\alpha_{2}} y_{s}}{d t^{\alpha_{2}}} \\
\frac{d^{\alpha_{3}} z_{s}}{d t^{\alpha_{3}}}
\end{array}\right)=\left(\begin{array}{ccc}
-a & a & 0 \\
c-a & c & 0 \\
0 & 0 & -b
\end{array}\right)\left(\begin{array}{l}
x_{s} \\
y_{s} \\
z_{s}
\end{array}\right) \\
& +\left(\begin{array}{c}
0 \\
-x_{m} z_{m} \\
x_{m} y_{m}
\end{array}\right)+\left(\begin{array}{l}
u_{1}(t) \\
u_{2}(t) \\
u_{3}(t)
\end{array}\right)
\end{aligned}
$$

where the controller $\mathbf{u}(t)=\left[u_{1}(t), u_{2}(t), u_{3}(t)\right]^{T}$ is defined in (15). Then the error system gives,

$$
\frac{d^{\alpha}}{d t^{\alpha}} \mathbf{e}(t)=B_{u} \mathbf{e}(t)
$$

where $\mathbf{e}(t)=\left(e_{1}(t), e_{2}(t), e_{3}(t)\right)$ and,

$$
B_{u}=\left(\begin{array}{ccc}
a_{11}-a & a_{12}+a & a_{13} \\
a_{21}+c-a & a_{22}+c & a_{23} \\
a_{31} & a_{32} & a_{33}-b
\end{array}\right) .
$$

In view of Case 1, if $\alpha_{1}=\alpha_{2}=\alpha_{3}=\alpha$, then the drive system (18) and the response system (19) are synchronized if all roots of the equation $\operatorname{det}\left(\lambda I-B_{u}\right)=0$ lie in the region $|\arg (\lambda)|>\alpha \pi / 2$, see Examples 1 and 2 below.

Also, in view of Case 2, if $\alpha_{1}=k_{1} / m_{1}, \alpha_{2}=$ $k_{2} / m_{2}$ and $\alpha_{3}=k_{3} / m_{3}$ are rational numbers, then both systems are synchronized if all roots of the equation $\operatorname{det}\left(\operatorname{diag}\left(\lambda^{r_{1}}, \lambda^{r_{2}}, \lambda^{r_{3}}\right)-B_{u}\right)=0$ lie in the region $|\arg (\lambda)|>\pi / 2 m$, where $m$ is the least common multiple of $\left\{m_{1}, m_{2}, m_{3}\right\}$ and $r_{i}=m \alpha_{i}$, see Example 3 below.
Moreover, in particular and according to Case 3, as in Examples 3 and 4 below, if $\alpha_{1}, \alpha_{2}, \alpha_{3}$ are any positive real numbers between 0 and 1 , then synchronization of the drive system (18) and the response system (19) is sufficiently achieved under the control laws,

$$
\left\{\begin{array}{l}
a_{12}=-a \\
a_{13}=0 \\
a_{23}=0 \\
a_{11}<a \\
a_{22}<-c \\
a_{33}<b
\end{array}\right.
$$

or,

$$
\left\{\begin{array}{l}
a_{21}=a-c \\
a_{31}=0 \\
a_{32}=0 \\
a_{11}<a \\
a_{22}<-c \\
a_{33}<b
\end{array}\right.
$$

Example 1. Taking $\alpha=(0.95,0.95,0.95),(a, b, c)=$ $(35,3,28)$ and $\mathbf{u}(t)=[(37,-33,0),(2,-31,0),(0,0$, $-5)] \mathbf{e}(t)$, then the roots of the equation $\operatorname{det}(\lambda I-$ $\left.B_{u}\right)=0$ are,

$$
\begin{aligned}
& \lambda_{1}=-5, \\
& \lambda_{2}=\frac{1}{2}(-1+\sqrt{15} i), \\
& \lambda_{3}=\frac{1}{2}(-1-\sqrt{15} i),
\end{aligned}
$$

where $i^{2}=-1$. Of course, all roots $\lambda_{1}, \lambda_{2}, \lambda_{3}$ have negative real parts, and so they lie in the region $|\arg (\lambda)|>(0.95) \pi / 2$. Therefore, the drive system (18) and the response system (19) are synchronized. The error functions evolution, in this case, is shown in Fig. 5(a).

Example 2. If we take $\alpha=(0.9,0.9,0.9),(a, b, c)=$ $(35,3,28)$ and $\mathbf{u}(t)=[(32,-35,0),(7,-26,-20)$, $(0,30,2)] \mathbf{e}(t)$, then the roots of the equation $\operatorname{det}\left(\lambda I-B_{u}\right)=0$ are,

$$
\begin{aligned}
& \lambda_{1}=-3, \\
& \lambda_{2}=\frac{1}{2}(1+\sqrt{2391} i), \\
& \lambda_{3}=\frac{1}{2}(1-\sqrt{2391} i) .
\end{aligned}
$$




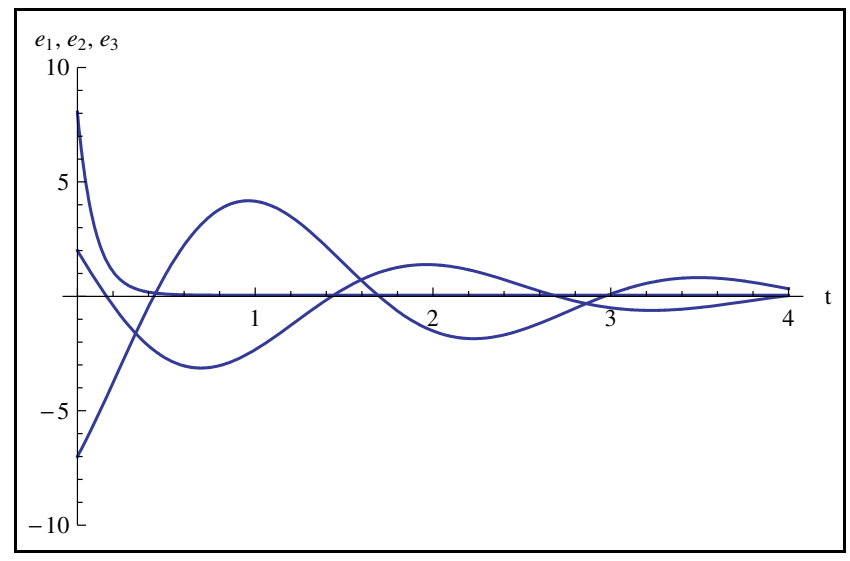

(a)

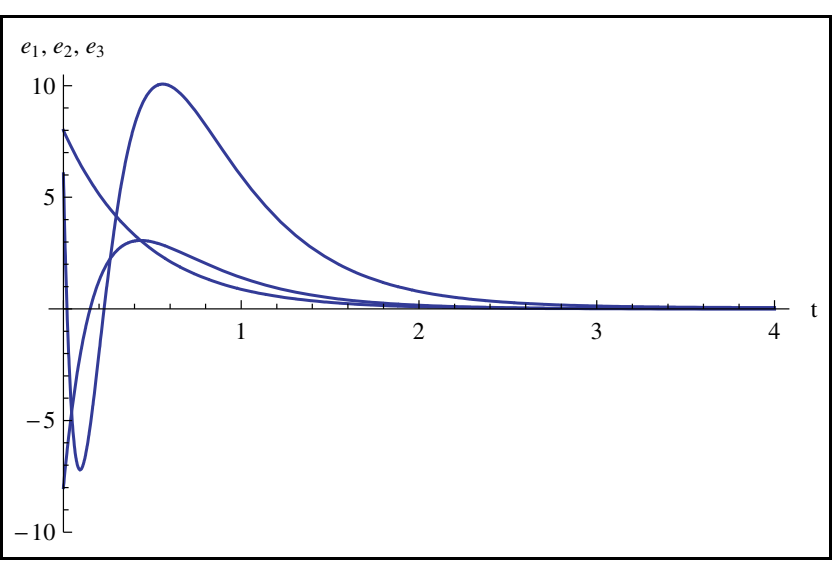

(c)

Fig. 5. Synchronization of the fractional-order Chen system (17). It is obvious that the evolution of the error functions $e_{1}(t), e_{2}(t), e_{3}(t) \rightarrow 0$ as $t \rightarrow+\infty$.

Even the eigenvalues $\lambda_{2}$ and $\lambda_{3}$ have positive real parts, systems (18) and (19) are synchronized since $\left|\arg \left(\lambda_{2,3}\right)\right|>(0.9) \pi / 2$. The error functions evolution, in this case, is shown in Fig. 5(b).

Example 3. If we take $\alpha=(0.985,0.99,0.98),(a$, $b, c)=(35,3,28)$ and $\mathbf{u}(t)=[(25,0,0),(7,-33,5)$, $(0,0,1)] \mathbf{e}(t)$, we have $r_{1}=985, r_{2}=990$, $r_{3}=980$ and $m=1000$ and then the equation $\operatorname{det}\left(\operatorname{diag}\left(\lambda^{985}, \lambda^{990}, \lambda^{980}\right)-B_{u}\right)=0$ can be written as,

$$
\left(\lambda^{985}+10\right)\left(\lambda^{990}+5\right)\left(\lambda^{980}+2\right)=0 .
$$

Using simple calculations, we can show that all roots of the above equation lie in the region $|\arg (\lambda)|>\pi / 2000$. Therefore, both systems (18)

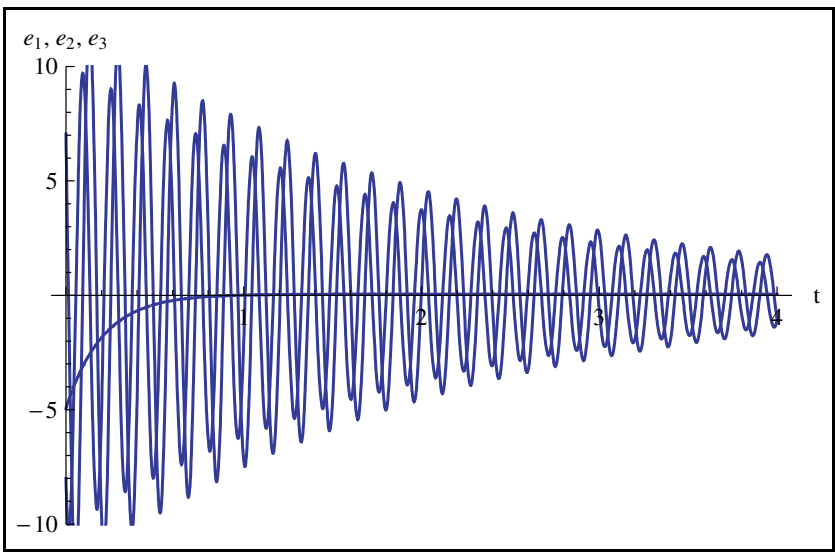

(b)

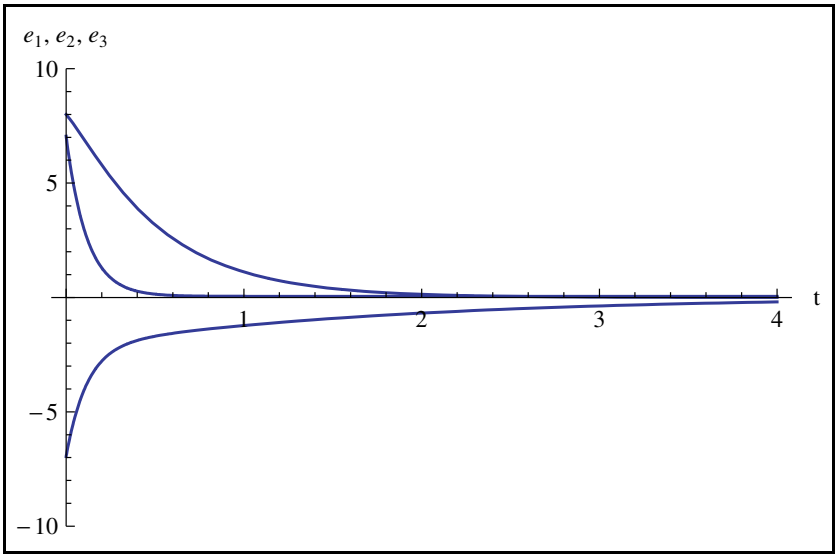

(d)

and (19) are synchronized. Also, we can conclude that the systems are synchronized since the controller $\mathbf{u}(t)$ satisfies the control law (22). The error functions evolution, in this case, is shown in Fig. 5(c).

Example 4. If we take $\alpha=(0.985,0.99,0.98),(a$, $b, c)=(35,3,28)$ and $\mathbf{u}(t)=[(27,-35,0),(8,-30$, $0),(5,0,2.5)] \mathbf{e}(t)$, then $\mathbf{u}(t)$ satisfies the control law (21), and so both systems (18) and (19) are synchronized. The error functions evolution, in this case, is shown in Fig. 5(d).

From Fig. 5, it is obvious that the components of the error system (20) decay towards zero as $t$ goes to $+\infty$. Therefore, we can numerically conclude that the designed linear controller can effectively control the chaotic fractional-order Chen system to 
achieve synchronization between the drive system (18) and the response system (19).

\section{Synchronization of \\ Fractional-Order Rössler Systems}

Now, we consider the Rössler system, designed by Rössler in 1976. Its fractional version reads as,

$$
\left\{\begin{array}{l}
\frac{d^{\alpha_{1}} x}{d t^{\alpha_{1}}}=-(y+z) \\
\frac{d^{\alpha_{2}} y}{d t^{\alpha_{2}}}=x+a y \\
\frac{d^{\alpha_{3}} z}{d t^{\alpha_{3}}}=z(x-c)+b
\end{array}\right.
$$

where $\alpha=\left(\alpha_{1}, \alpha_{2}, \alpha_{3}\right)$ is subject to $0<\alpha_{1}, \alpha_{2}, \alpha_{3} \leq$ 1 . When $\alpha=(1,1,1)$, system (23) is the original integer order Rössler system, which is chaotic, for example, when $(a, b, c)=(0.2,0.2,5)$, see Fig. 6. Simulations are performed to obtain chaotic behavior of the fractional-order Rössler system and the results demonstrate that chaos indeed exists with order less than 3 (i.e. $\alpha_{1}+\alpha_{2}+\alpha_{3}<$ $3)$. For example, chaotic attractors are found in [Li \& Chen, 2004a] when $\alpha=(0.9,0.9,0.9)$ and $(a, b, c)=(0.4,0.2,10)$. Also, in [Peng, 2007] it is found that for the parameters $\alpha=(1,1,0.8)$ and $(a, b, c)=(0.2,0.2,5)$ the fractional-order Rössler system can display chaotic behaviors. Moreover, we find that chaotic behaviors can be displayed for the fractional-order Rössler system when $(a, b, c)=$ $(0.2,0.2,5)$ and $\alpha=(0.95,0.95,0.95)$ or $\alpha=$ $(0.9,0.9,0.9)$, see Figs. 7 and 8.

According to our approach, the drive and the response fractional-order Rössler systems are described as,

$$
\begin{aligned}
\left(\begin{array}{c}
\frac{d^{\alpha_{1}} x_{m}}{d t^{\alpha_{1}}} \\
\frac{d^{\alpha_{2}} y_{m}}{d t^{\alpha_{2}}} \\
\frac{d^{\alpha_{3}} z_{m}}{d t^{\alpha_{3}}}
\end{array}\right)= & \left(\begin{array}{ccc}
0 & -1 & -1 \\
1 & a & 0 \\
0 & 0 & -c
\end{array}\right)\left(\begin{array}{l}
x_{m} \\
y_{m} \\
z_{m}
\end{array}\right) \\
& +\left(\begin{array}{c}
0 \\
0 \\
x_{m} z_{m}+b
\end{array}\right)
\end{aligned}
$$
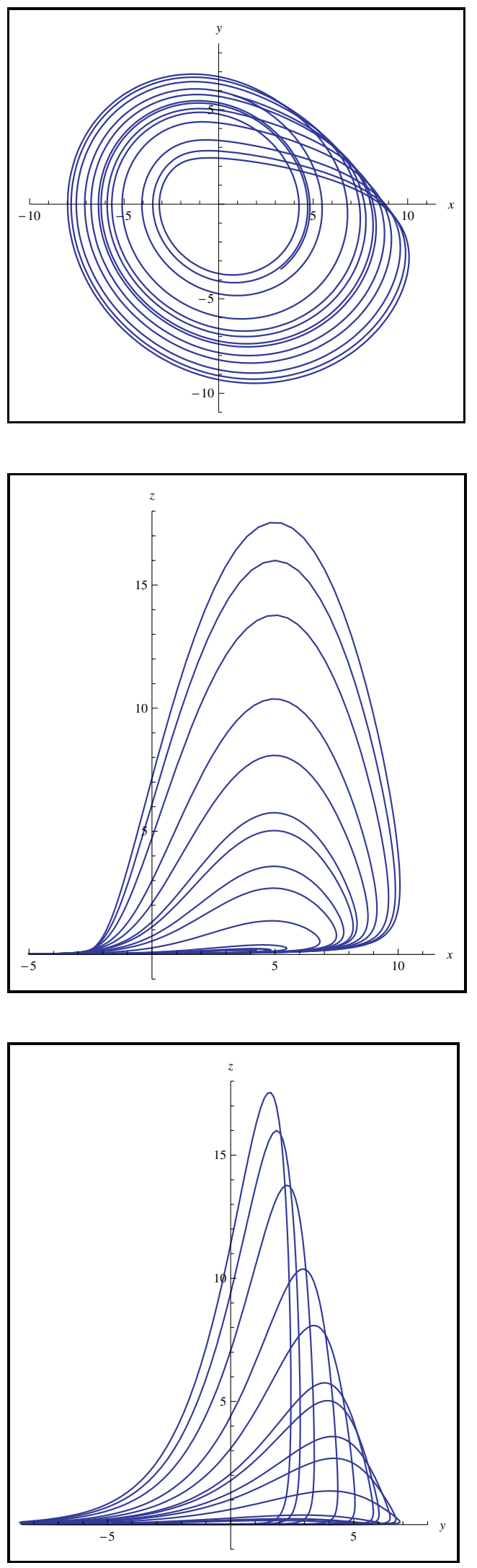

Fig. 6. Chaotic attractor of the Rössler system (23), when $\alpha=(1,1,1)$ and $(a, b, c)=(0.2,0.2,5)$. 
Synchronization of Chaotic Fractional-Order Systems via Linear Control 91
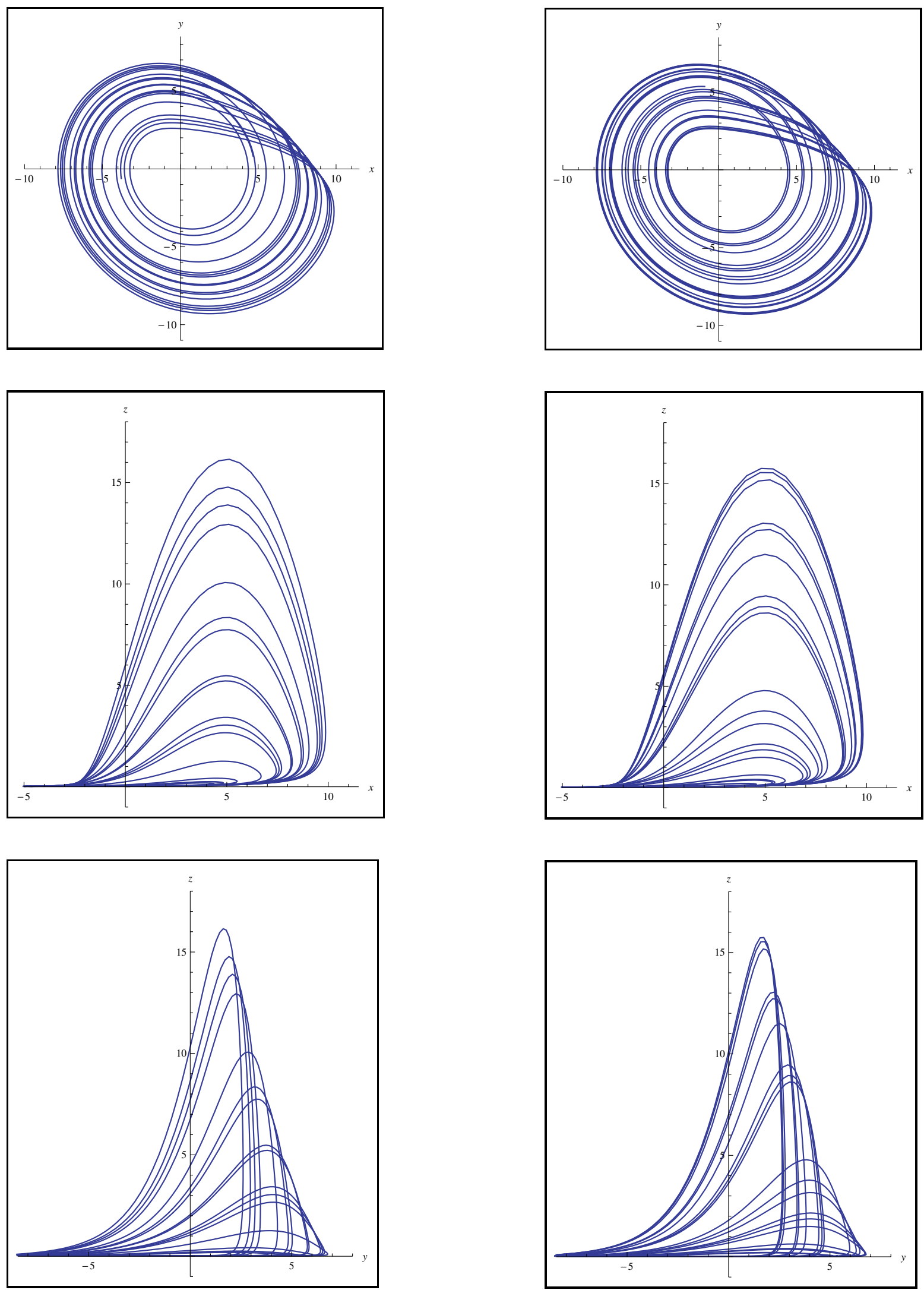

Fig. 7. Chaotic attractor of the Rössler system (23), when $\alpha=(0.95,0.95,0.95)$ and $(a, b, c)=(0.2,0.2,5)$.

Fig. 8. Chaotic attractor of the Rössler system (23), when $\alpha=(0.9,0.9,0.9)$ and $(a, b, c)=(0.2,0.2,5)$. 


$$
\begin{aligned}
\left(\begin{array}{c}
\frac{d^{\alpha_{1}} x_{s}}{d t^{\alpha_{1}}} \\
\frac{d^{\alpha_{2}} y_{s}}{d t^{\alpha_{2}}} \\
\frac{d^{\alpha_{3}} z_{s}}{d t^{\alpha_{3}}}
\end{array}\right)= & \left(\begin{array}{ccc}
0 & -1 & -1 \\
1 & a & 0 \\
0 & 0 & -c
\end{array}\right)\left(\begin{array}{l}
x_{s} \\
y_{s} \\
z_{s}
\end{array}\right) \\
& +\left(\begin{array}{c}
0 \\
0 \\
x_{m} z_{m}+b
\end{array}\right)+\left(\begin{array}{l}
u_{1}(t) \\
u_{2}(t) \\
u_{3}(t)
\end{array}\right)
\end{aligned}
$$

where the controller $\mathbf{u}(t)=\left[u_{1}(t), u_{2}(t), u_{3}(t)\right]^{T}$ is defined in (15). Then the error system gives,

$$
\frac{d^{\alpha}}{d t^{\alpha}} \mathbf{e}(t)=C_{u} \mathbf{e}(t)
$$

where,

$$
C_{u}=\left(\begin{array}{ccc}
a_{11} & a_{12}-1 & a_{13}-1 \\
a_{21}+1 & a_{22}+a & a_{23} \\
a_{31} & a_{32} & a_{33}-c
\end{array}\right) .
$$

In view of Case 1, if $\alpha_{1}=\alpha_{2}=\alpha_{3}=\alpha$, then the drive system (24) and the response system (25) are synchronized if all roots of the equation $\operatorname{det}\left(\lambda I-C_{u}\right)=0$ lie in the region $|\arg (\lambda)|>\alpha \pi / 2$, see Example 5 below.

Also, in view of Case 2, if $\alpha_{1}=k_{1} / m_{1}, \alpha_{2}=$ $k_{2} / m_{2}$ and $\alpha_{3}=k_{3} / m_{3}$ are rational numbers, then both systems are synchronized if all roots of the equation $\operatorname{det}\left(\operatorname{diag}\left(\lambda^{r_{1}}, \lambda^{r_{2}}, \lambda^{r_{3}}\right)-C_{u}\right)=0$ lie in the region $|\arg (\lambda)|>\pi / 2 m$, where $m$ is the least common multiple of $\left\{m_{1}, m_{2}, m_{3}\right\}$ and $r_{i}=m \alpha_{i}$, see Example 6.

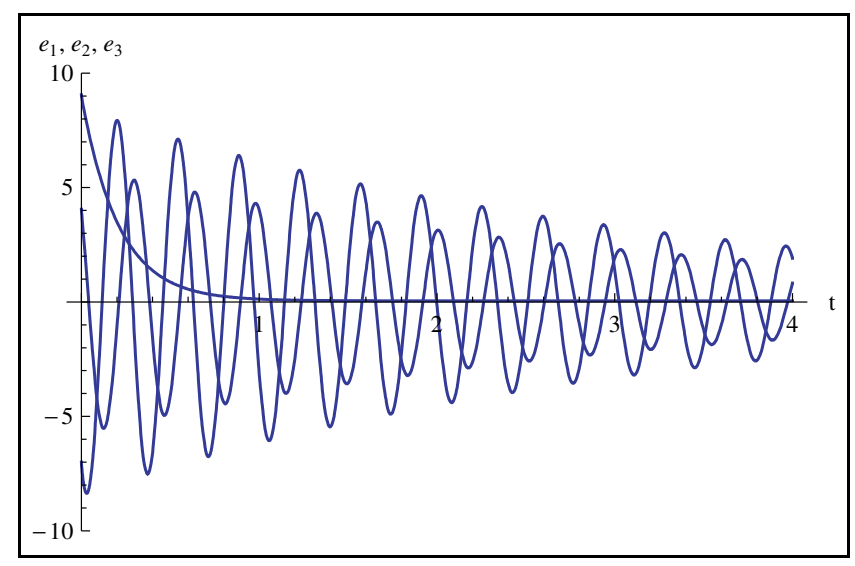

(a)
Moreover, in particular and according to Case 3 , if $\alpha_{1}, \alpha_{2}, \alpha_{3}$ are any positive real numbers between 0 and 1 , then synchronization of the drive system (24) and the response system (25) is sufficiently achieved under the control laws,

$$
\left\{\begin{array}{l}
a_{12}=-1 \\
a_{13}=1 \\
a_{23}=0 \\
a_{11}<0 \\
a_{22}<-a \\
a_{33}<c
\end{array}\right.
$$

or,

$$
\left\{\begin{array}{l}
a_{21}=-1 \\
a_{31}=0 \\
a_{32}=0 \\
a_{11}<0 \\
a_{22}<-a \\
a_{33}<c
\end{array}\right.
$$

Example 5. Taking $\alpha=(0.9,0.9,0.9),(a, b, c)=$ $(0.4,0.2,10)$ and $\mathbf{u}(t)=[(2,9,1),(-18,-2.4,0)$, $(0,0,7)] \mathbf{e}(t)$, then the roots of the equation $\operatorname{det}(\lambda I-$ $\left.C_{u}\right)=0$ are,

$$
\lambda_{1}=-3, \quad \lambda_{2}=\sqrt{132} i, \quad \lambda_{3}=-\sqrt{132} i .
$$

Since $\left|\arg \left(\lambda_{1,2,3}\right)\right|>(0.9) \pi / 2$, the drive system $(24)$ and the response system (25) are synchronized. The error functions evolution, in this case, is shown in Fig. 9(a).

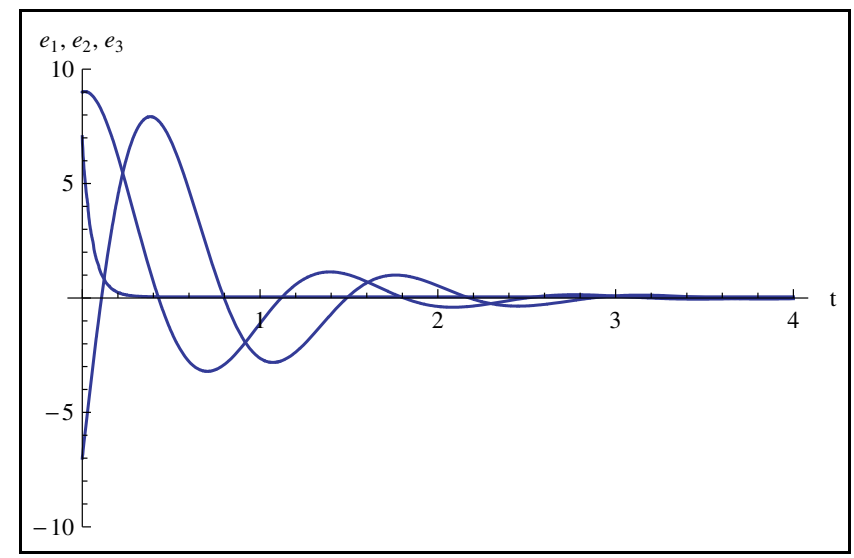

(b)

Fig. 9. Synchronization of the fractional-order Rössler system (23). It is obvious that the evolution of the error functions $e_{1}(t), e_{2}(t), e_{3}(t) \rightarrow 0$ as $t \rightarrow+\infty$. 
Example 6. If we take $\alpha=(1,1,0.8),(a, b, c)=$ $(0.2,0.2,5)$ and $\mathbf{u}(t)=[(-2,-2,1),(6,-1.2,0)$, $(0,0,-2)] \mathbf{e}(t)$, we have $r_{1}=10, r_{2}=10$, $r_{3}=8$ and $m=10$ and then the equation $\operatorname{det}\left(\operatorname{diag}\left(\lambda^{10}, \lambda^{10}, \lambda^{8}\right)-C_{u}\right)=0$ can be written as,

$$
\left(\lambda^{8}+2\right)\left(\lambda^{20}+3 \lambda^{10}+20\right)=0 .
$$

Simply, we can show that all roots of the above equation lie in the region $|\arg (\lambda)|>\pi / 20$. Therefore, both systems (24) and (25) are synchronized. The error functions evolution, in this case, is shown in Fig. 9(b).

From Fig. 9, for the given parameters, we can conclude that the components of the error system (26) decay towards zero as $t$ goes to $+\infty$, and so the drive system (24) and the response system (25) are synchronized.

\section{Synchronization of Fractional-Order Cubic Chua Systems}

Now, we consider Chua's equations [Chua \& Lin, 1990; Chua et al., 1993] with cubic nonlinearity (i.e. cubic Chua system), see for example [Huang et al., 1996]. Its fractional version reads as,

$$
\left\{\begin{array}{l}
\frac{d^{\alpha_{1}} x}{d t^{\alpha_{1}}}=a\left(y+b x+c x^{3}\right) \\
\frac{d^{\alpha_{2}} y}{d t^{\alpha_{2}}}=x-y+z \\
\frac{d^{\alpha_{3}} z}{d t^{\alpha_{3}}}=-\beta y-\gamma z
\end{array}\right.
$$

where $\alpha=\left(\alpha_{1}, \alpha_{2}, \alpha_{3}\right)$ is subject to $0<\alpha_{1}, \alpha_{2}, \alpha_{3} \leq$ 1. We fix the parameters as,

$$
(a, b, c)=(9.5,0.15,-0.3), \quad(\beta, \gamma)=(14,0.02)
$$

and we perform numerical simulations to obtain chaotic behaviors of the fractional-order cubic Chua system. First of all, the integer case $\alpha=(1,1,1)$ is studied and chaotic attractors are found as shown in Fig. 10. Then, in the fractional-order case, with $\alpha=$ $(0.98,0.98,0.98)$ or $\alpha=(0.96,0.96,0.96)$, chaotic behaviors are also obtained as shown in Figs. 11 and 12 .
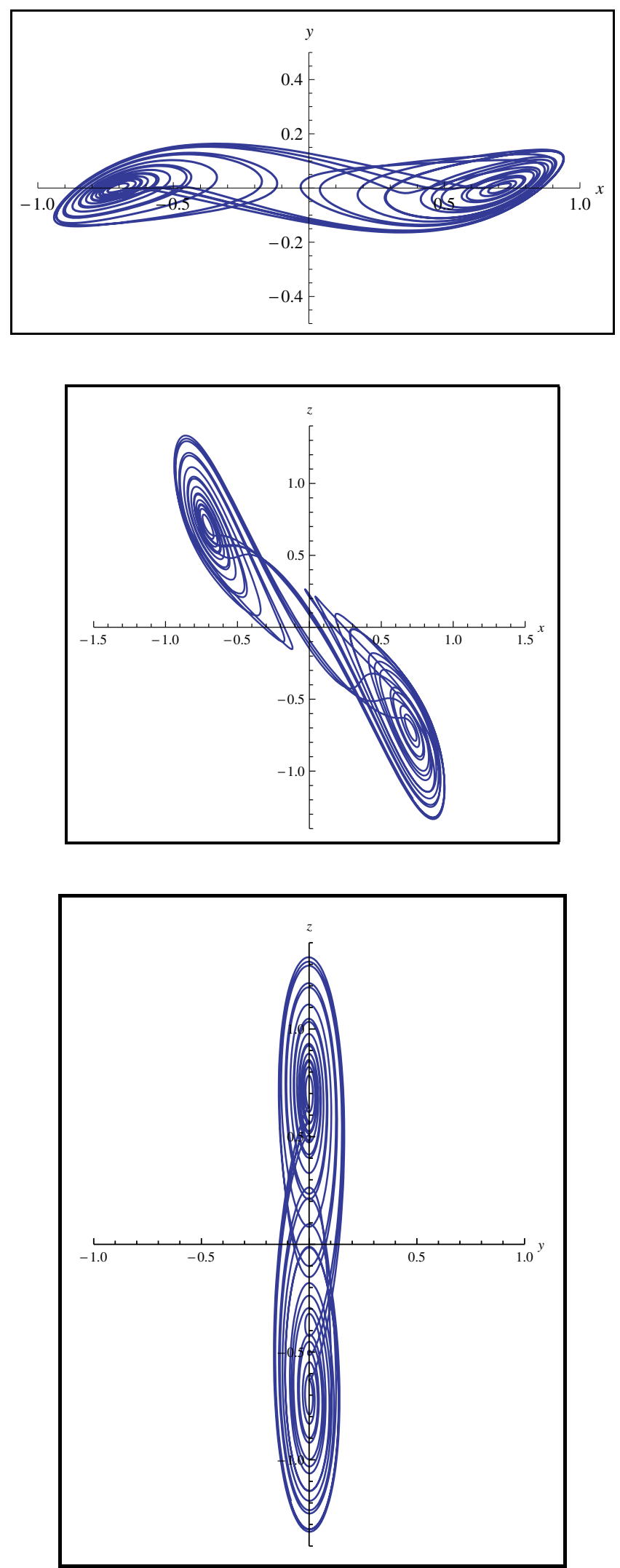

Fig. 10. Chaotic attractor of the cubic Chua system (29), when $\alpha=(1,1,1)$ with the parameters as given in Eq. (30). 


$$
\begin{aligned}
& 57 \\
& 11
\end{aligned}
$$


According to our approach, the drive and the response systems for the fractional-order cubic Chua system are described as,

$$
\begin{aligned}
\left(\begin{array}{l}
\frac{d^{\alpha_{1}} x_{m}}{d t^{\alpha_{1}}} \\
\frac{d^{\alpha_{2}} y_{m}}{d t^{\alpha_{2}}} \\
\frac{d^{\alpha_{3}} z_{m}}{d t^{\alpha_{3}}}
\end{array}\right)= & \left(\begin{array}{ccc}
-a b & a & 0 \\
1 & -1 & 1 \\
0 & -\beta & -\gamma
\end{array}\right)\left(\begin{array}{l}
x_{m} \\
y_{m} \\
z_{m}
\end{array}\right) \\
& +\left(\begin{array}{cc}
-a c x_{m}^{3} \\
0 \\
0
\end{array}\right) \\
\left(\begin{array}{c}
\frac{d^{\alpha_{1}} x_{s}}{d t^{\alpha_{1}}} \\
\frac{d^{\alpha_{2}} y_{s}}{d t^{\alpha_{2}}} \\
\frac{d^{\alpha_{3}} z_{s}}{d t^{\alpha_{3}}}
\end{array}\right)= & \left(\begin{array}{ccc}
-a b & a & 0 \\
1 & -1 & 1 \\
0 & -\beta & -\gamma
\end{array}\right)\left(\begin{array}{l}
x_{s} \\
y_{s} \\
z_{s}
\end{array}\right) \\
& +\left(\begin{array}{c}
-a c x_{m}^{3} \\
0 \\
0
\end{array}\right)+\left(\begin{array}{l}
u_{1}(t) \\
u_{2}(t) \\
u_{3}(t)
\end{array}\right)
\end{aligned}
$$

Then, the error system gives,

$$
\frac{d^{\alpha}}{d t^{\alpha}} \mathbf{e}(t)=D_{u} \mathbf{e}(t)
$$

where,

$$
D_{u}=\left(\begin{array}{ccc}
a_{11}-a b & a_{12}+a & a_{13} \\
a_{21}+1 & a_{22}-1 & a_{23}+1 \\
a_{31} & a_{32}-\beta & a_{33}-\gamma
\end{array}\right)
$$

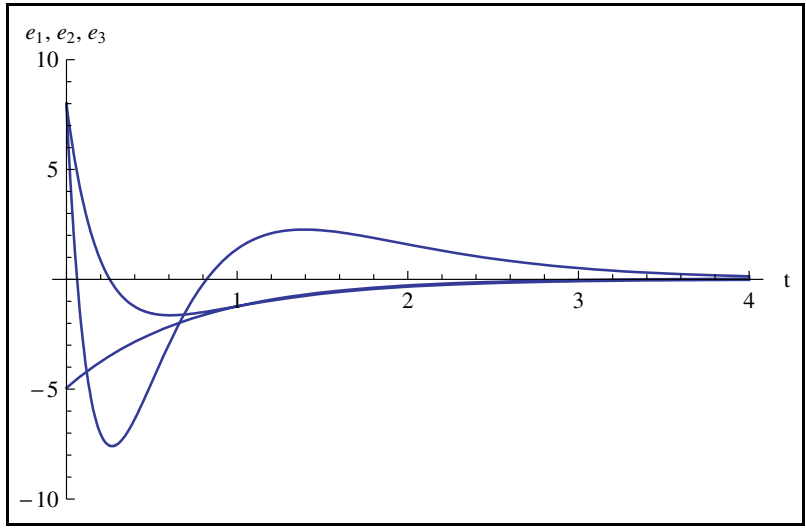

(a)
Similar to the previous systems, in view of Case 1 , if $\alpha_{1}=\alpha_{2}=\alpha_{3}=\alpha$, then the drive system (31) and the response system (32) are synchronized if all roots of the equation $\operatorname{det}\left(\lambda I-D_{u}\right)=0$ lie in the region $|\arg (\lambda)|>\alpha \pi / 2$.

Also, in view of Case 2, if $\alpha_{1}=k_{1} / m_{1}, \alpha_{2}=$ $k_{2} / m_{2}$ and $\alpha_{3}=k_{3} / m_{3}$ are rational numbers, then both systems are synchronized if all roots of the equation $\operatorname{det}\left(\operatorname{diag}\left(\lambda^{r_{1}}, \lambda^{r_{2}}, \lambda^{r_{3}}\right)-D_{u}\right)=0$ lie in the region $|\arg (\lambda)|>\pi / 2 m$, where $m$ is the least common multiple of $\left\{m_{1}, m_{2}, m_{3}\right\}$ and $r_{i}=m \alpha_{i}$.

Moreover, in particular and according to Case 3, as in Examples 7 and 8, if $\alpha_{1}, \alpha_{2}, \alpha_{3}$ are any positive real numbers between 0 and 1, then synchronization of the drive system (31) and the response system (32) is sufficiently achieved under the control laws,

$$
\left\{\begin{array}{l}
a_{12}=-a \\
a_{13}=0 \\
a_{23}=-1 \\
a_{11}<a b \\
a_{22}<1 \\
a_{33}<\gamma
\end{array}\right.
$$

or,

$$
\left\{\begin{array}{l}
a_{21}=-1 \\
a_{31}=0 \\
a_{32}=\beta \\
a_{11}<a b \\
a_{22}<1 \\
a_{33}<\gamma
\end{array}\right.
$$

Example 7. Taking $\alpha=(0.98,0.98,0.98)$, the same parameters as given in Eq. (30) and

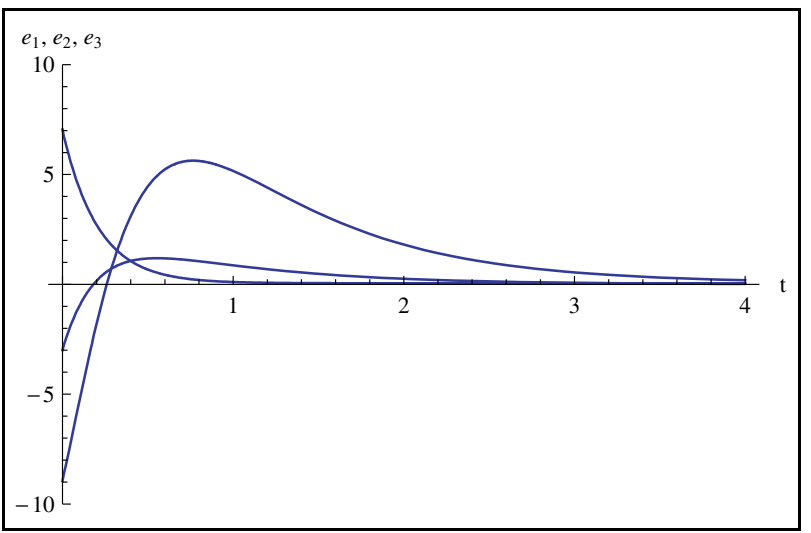

(b)

Fig. 13. Synchronization of the fractional-order cubic Chua system (29). It is obvious that the evolution of the error functions $e_{1}(t), e_{2}(t), e_{3}(t) \rightarrow 0$ as $t \rightarrow+\infty$. 
$\mathbf{u}(t)=[(-2.5,-9.5,0),(2,-3,-1),(5,3,-2)] \mathbf{e}(t)$, then $\mathbf{u}(t)$ satisfies the control law (34), and so both systems (31) and (32) are synchronized. The error functions evolution, in this case, is shown in Fig. 13(a).

Example 8. If we take $\alpha=(0.96,0.96,0.96)$, the same parameters as given in Eq. (30) and $\mathbf{u}(t)=[(-4,3,5),(-1,0.2,1),(0,14,-3)] \mathbf{e}(t)$, then $\mathbf{u}(t)$ satisfies the control law (35), and so both systems (31) and (32) are synchronized. The error functions evolution, in this case, is shown in Fig. 13(b).

From Fig. 13, for the given parameters, we can conclude that the components of the error system (33) decay towards zero as $t \rightarrow+\infty$, and so the drive system (31) and the response system (32) are synchronized.

\section{Conclusion}

In this paper, we present theoretical results for drive-response synchronization between fractionalorder systems, based on stability results of linear fractional-order systems. Suitable sufficient conditions for achieving synchronization of two identical fractional-order systems via a suitable linear controller applied to the response system are derived. We briefly discuss the issue of controlling fractionalorder chaotic Chen, Rössler and modified Chua systems to realize synchronization with linear error feedback control. For this purpose, we design the controller such that the components of the error system decay towards zero as $t$ goes to $+\infty$. Numerical simulations are given to verify the effectiveness of the proposed synchronization scheme and to show that some typical chaotic systems can be synchronized. We emphasize that this scheme of synchronization can be extended to other chaotic fractional-order systems.

\section{References}

Arneodo, A., Coullet, P., Spiegel, E. \& Tresser, C. [1985] "Asymptotic chaos," Physica D 14, 327-347.

Aziz-Alaoui, M. A. [2006] "Synchronization of chaos," Encyclopedia of Mathematical Physics (Elsevier), pp. 213-226.

Caputo, M. [1967] "Linear models of dissipation whose $Q$ is almost frequency independent, Part II," J. Roy. Astr. Soc. 13, 529-539.

Chen, C. \& Ueta, T. [1999] "Yet another chaotic attractor," Int. J. Bifurcation and Chaos 9, 1465-1466.
Chen, G. \& Dong, X. [1998] From Chaos to Order: Methodologies, Persectives and Applications (World Scientific, Singapore).

Chua, L. O. \& Lin, G.-N. [1990] "Canonical realization of Chua's circuit family," IEEE Trans. Circuits Syst. 37, 885-902.

Chua, L. O., Makoto, I., Kocarev, L. \& Eckert, K. [1993] "Chaos synchronization in Chua's circuit," J. Circuits Syst. Comput. 3, 93-108.

Deng, W. H. \& Li, C. P. [2005] "Chaos synchronization of the fractional Lü system," Physica A 353, 61-72.

Deng, W., Li, C. \& Lü, J. [2007] "Stability analysis of linear fractional differential system with multiple time delays," Nonlin. Dyn. 48, 409-416.

Deriviěre, S. \& Aziz-Alaoui, M. A. [2003] "Estimation of attractors and synchronization of generalized Lorenz systems," Dyn. Contin. Discr. Impul. Syst. Series B: Appl. Algorith. 10, 833-852.

Ge, Z. M. \& Ou, C. Y. [2007] "Chaos in a fractional order modified Duffing system," Chaos Solit. Fract. 34, 262-291.

Gorenflo, R. \& Mainardi, F. [1997] "Fractional calculus: Integral and differential equations of fractional order," Fractals and Fractional Calculus, eds. Carpinteri, A. \& Mainardi, F., pp. 223-276.

Grigorenko, I. \& Grigorenko, E. [2003] "Chaotic dynamics of the fractional Lorenz system," Phys. Rev. Lett. 91, 034101 .

Hartley, T., Lorenzo, C. \& Qammer, H. [1995] "Chaos in a fractional order Chua's system," IEEE Trans. CASI 42, 485-490.

Hilfer, R. [2000] Applications of Fractional Calculus in Physics (World Scientific, NJ).

Huang, A., Pivka, L. \& Wu, C. W. [1996] "Chua's equation with cubic nonlinearity," Int. J. Bifurcation and Chaos 6, 2175-2222.

Li, C. \& Chen, G. [2004a] "Chaos and hyperchaos in fractional order Rössler equations," Physica A 341, $55-61$.

Li, C. \& Chen, G. [2004b] "Chaos in the fractional order Chen system and its control," Chaos Solit. Fract. 22, 549-554.

Li, C. \& Peng, G. [2004] "Chaos in Chen's system with a fractional order," Chaos Solit. Fract. 22, 443450.

Li, C. \& Zhou, T. [2005] "Synchronization in fractionalorder differential systems," Physica D 212, 111-125.

Li, C. \& Yan, J. [2007] "The synchronization of three fractional differential systems," Chaos Solit. Fract. 32, 751-757.

Li, C. G., Liao, X. X. \& Yu, J. B. [2003] "Synchronization of fractional order chaotic systems," Phys. Rev. E 68, 067203.

Li, C. P., Deng, W. H. \& Xu, D. [2006] "Chaos synchronization of the Chua system with a fractional order," Physica A 360, 171-185. 
Lu, J. G. \& Chen, G. [2006] "A note on the fractionalorder Chen system," Chaos Solit. Fract. 27, 685688.

Matignon, D. [1996] "Stability results of fractional differential equations with applications to control processing," in Proc. IMACS, IEEE-SMC, Lille, France, pp. 963-968.

Oldham, K. B. \& Spanier, J. [1974] The Fractional Calculus (Academic Press, NY).

Ott, E., Grebogi, C. \& Yorke, J. A. [1990] "Controling chaos," Phys. Rev. Lett. 64, 1196-1199.

Pecora, L. M. \& Carrol, T. L. [1990] "Synchronization in chaotic systems," Phys. Rev. Lett. 64, 821-824.

Peng, G. [2007] "Synchronization of fractional order chaotic systems," Phys. Lett. A 363, 426-432.

Petráš, I. [2008] "A note on the fractional-order Chua's system," Chaos Solit. Fract. 38, 140-147.

Podlubny, I. [1999] Fractional Differential Equations (Academic Press, NY).

Rössler, O. E. [1976] "An equation for continuous chaos," Phys. Lett. A 57, 397-398.

Sheu, L. J., Chen, H. K., Chen, J. H. \& Tam, L. M. [2007] "Chaos in a new system with fractional order," Chaos Solit. Fract. 31, 1203-1212.
Wang, J., Xiong, X. \& Zhang, Y. [2006] "Extending synchronization scheme to chaotic fractional-order Chen systems," Physica A 370, 279-285.

Wu, X., Li, J. \& Chen, G. J. [2008] "Chaos in the fractional order unified system and its synchronization," J. Franklin Instit. 345, 392-401.

Yamada, T. \& Fujisaka, H. [1983] "Stability theory of synchroized motion in coupled-oscillator systems," Prog. Theoret. Phys. 70, 1240-1248.

Yan, J. \& Li, C. [2007] "On chaos synchronization of fractional differential equations," Chaos Solit. Fract. 32, 725-735.

Yu, Y. \& Li, H. [2008] "The synchronization of fractional-order Rössler hyperchaotic systems," Physica A 387, 1393-1403.

Zhou, S., Li, H., Zhu, Z. \& Li, C. [2008] "Chaos control and synchronization in a fractional neuron network system," Chaos Solit. Fract. 36, 973-984.

Zhu, H., Zhou, S. \& Zhang, J. [2009] "Chaos and synchronization of the fractional-order Chua's system," Chaos Solit. Fract. 39, 1595-1603. 